\title{
INTERAÇÃO CULTIVAR DE FEIJÃO E CONDIÇÕES ATMOSFÉRICAS SOBRE Callosobruchus maculatus (COLEOPTERA: BRUCHIDAE) EM GRÃOS ARMAZENADOS
}

\author{
Francisco Roberto Azevedo ${ }^{1}$ e Maria Andréia Moura ${ }^{2}$ \\ ${ }^{1}$ Laboratório de Entomologia da Universidade Federal do Ceará - Campus Cariri \\ razevedo@ufc.br - andreiamoura.kd@bol.com.br
}

Artigo submetido em dezembro/2012 e aceito em dezembro/2013

\section{RESUMO}

Objetivando avaliar a interação de uma cultivar de feijão suscetível comparada a uma não hospedeira armazenada em três condições atmosféricas: hermética, semi hermética e não hermética, sobre o desenvolvimento do caruncho Callosobruchus maculatus (L.), conduziu-se essa pesquisa no laboratório de Entomologia da UFC Campus Cariri, no Crato-CE, em condições ambiente, durante maio a julho de 2008. Utilizou-se o delineamento inteiramente casualizado, com quatro repetições em esquema fatorial $2 \times 3$, donde o primeiro fator referiu-se as cultivares de feijão e o segundo fator, as condições atmosféricas. Aos oito dias de infestação, avaliou-se a mortalidade dos adultos e a oviposição e, no final do período de armazenamento, que correspondeu aos quarenta e cinco dias, foram avaliadas a emergência dos carunchos e a perda de peso dos grãos. A condição hermética promove maior mortalidade e menor emergência dos adultos do caruncho, assim como, menor perda de peso nos grãos. Portanto, a interação de uma cultivar suscetível de feijão armazenada em uma condição hermética pode ser utilizada como uma tática alternativa para o agricultor familiar ao uso do controle químico em programas de manejo de Callosobruchus maculatus em grãos armazenados.

PALAVRAS-CHAVE: caruncho, controle hermético, caupi, pragas de grãos armazenados.

\section{INTERACTIONS BETWEEN CULTIVATE BEAN AND ATMOSPHERIC CONDITIONS ABOUT Callosobruchus maculatus (COLEOPTERA: BRUCHIDAE) ON STORED GRAIN.}

\begin{abstract}
Objectifying to evaluate the interaction of a susceptible bean cultivar compared with no host bean cultivar stored in three atmospheric conditions: hermetic, semihermetic and no hermetic, about the development of weevil Callosobruchus maculatus (L.), conducted this research in the laboratory of Entomology of the UFC, in Crato-CE, on ambient conditions, during may to july of 2008. It was utilized a completely randomized design, with four replicates in a factorial $2 \times 3$, where the first factor referred to the cultivars of beans and as other factor, the three atmospheric conditions. At the eight
\end{abstract}

days of infestation were evaluated the mortality of the adults and the egg-laying and, in the end of the storage period, evaluated the emergency of weevil and the loss of weight of the grains. The condition hermetic promotes higher mortality and lower emergency of the adults of weevil, along, lower loss of weight of the grains. Therefore, the hermetic control associated with susceptible cultivars of beans can be used as strategic alternatives for small growers to the use of chemical products in management programs of Callosobruchus maculatus in stored grains.

KEYWORDS: bean weevil, hermetic control, cowpea, pests stored grains. 


\section{INTERAÇÃO CULTIVAR DE FEIJÃO E CONDIÇÕES ATMOSFÉRICAS SOBRE Callosobruchus maculatus (COLEOPTERA: BRUCHIDAE) EM GRÃOS ARMAZENADOS}

\section{INTRODUÇÃO}

O caupi, Vigna unguiculata (L.) Walp, é uma das principais leguminosas de grãos cultivadas nas regiões tropicais e subtropicais, sendo utilizados na alimentação humana como fonte de proteínas, carboidratos, cálcio, ferro e vitaminas (MACEDO et al., 2002).

O caruncho Callosobruchus maculatus (Fabr. 1775) (Coleoptera: Bruchidae) ataca geralmente feijões do gênero Vigna. Como no Nordeste do Brasil a maioria do feijão plantado e consumido pertence a esse gênero, esse caruncho é considerado a principal praga da região. 0 seu ataque inicia-se no campo, onde oviposita nas vagens deiscentes ou defeituosas, antes da colheita e intensifica-se nos grãos armazenados (GALLO et al., 2002).

É necessária a proteção contínua dos produtos armazenados contra a deterioração evitando-se dessa forma, perdas de qualidade e quantidade durante o armazenamento, objetivando-se atender a um mercado cada vez mais exigente (PADIN et al., 2002).

Em armazenamentos comerciais de caupi, o controle do $C$. maculatus geralmente tem sido realizado com o uso do fosfeto de alumínio, na dosagem de duas pastinhas de $3 \mathrm{~g} / \mathrm{m}^{3}$. No entanto, esse produto representa um grande risco para o armazenamento, já que são muitos os relatos que mostram a manifestação da resistência desses insetos a esse produto químico (MBATA et al., 2004).

Dentro das diferentes formas de minimizar o ataque das pragas e de diminuir a pressão de seleção de indivíduos resistentes aos produtos, a utilização de variedades resistentes possui a resistência contra insetos incorporada ao seu genoma, proporcionando menores custos de produção e não afetando o equilíbrio ambiental (GUZZO et al., 2002).

Cultivares resistentes têm atributos físicos ou bioquímicos que afetam o desenvolvimento ou a sobrevivência dos carunchos e o feijão por apresentar elevada variabilidade genética, pode ser melhorado para o controle de carunchos associado com outras técnicas de controle, como a modificação atmosférica (THRONE et al., 2000).

O controle do caruncho utilizando atmosfera modificada por alcançar o sistema respiratório do inseto em sua demanda por oxigênio e aproveitar o efeito nocivo da alta concentração de $\mathrm{CO}_{2}$ (FARONI et al. 2002) e baixas concentrações de oxigênio tem sido efetivo no controle de C. maculatus, conforme trabalhos realizados por Mbata e Phillips (2001), Ofuya e Reichmuth (2002), Shazali et al. (2004), Mbata et al. (2005; 2009).

Considerando os problemas que representa $C$. maculatus para o armazenamento de feijão e a sua capacidade em expressar resistência, realizou-se essa pesquisa com o objetivo de estudar a associação de uma cultivar suscetível de feijão em três condições atmosféricas sobre o desenvolvimento do caruncho em grãos armazenados em condições ambiente. 


\section{MATERIAL E MÉTODOS}

A pesquisa foi conduzida no laboratório de Entomologia da UFC Campus Cariri, no Crato$\mathrm{CE}$, em condições ambiente a uma temperatura média de $28,8 \pm 1{ }^{\circ} \mathrm{C}$, umidade relativa de $60,5 \pm$ $10 \%$ e fotofase de 14 horas, durante maio a julho de 2008.

Foram comparadas duas cultivares de feijão: Vigna unguiculata (L. Walp) cv. caupi (suscetível) e Phaseolus vulgaris (L.) cv. carioca (não hospedeira), comercializadas no estado do Ceará. Esses feijões foram armazenados nas condições atmosféricas: hermética, semi hermética e não hermética. Para obtenção dessas condições, os feijões foram armazenados em copos de acrílico transparentes com capacidade para $300 \mathrm{~mL}$ previamente esterilizados.

$\mathrm{Na}$ atmosfera hermética os copos foram tapados hermeticamente com isopor e lacrados com fita gomada de modo a não permitir a passagem de oxigênio para o interior dos copos.

$\mathrm{Na}$ atmosfera semi hermética, além dos copos estarem cobertos com isopor, que continham seis furos equidistantes com diâmetros de $1 \mathrm{~cm}$, fez-se necessário também a utilização de um tecido branco tipo organza sob o isopor, para evitar a fuga dos insetos, promovendo a passagem parcial de oxigênio.

Já na atmosfera não hermética, os copos foram apenas cobertos com a organza, permitindo assim, a livre passagem de oxigênio entre os grãos presentes no interior dos copos.

Depois do acondicionamento dos grãos nos copos, fez-se a infestação com 20 adultos não sexados do caruncho com idade variando de 0 a 72 horas, com o auxílio de um aspirador bucal, provenientes de uma criação estoque mantida no próprio laboratório.

Cada parcela experimental consistiu de 50 grãos e 20 insetos para cada cultivar de feijão e condição atmosférica. Foi utilizado o delineamento inteiramente casualizado, com 4 repetições em esquema fatorial $2 \times 3$, donde o primeiro fator se refere as cultivares de feijão e o segundo fator, as condições atmosféricas, totalizando assim, 24 parcelas em todo o experimento.

\subsection{Efeito sobre a mortalidade e oviposição dos adultos}

Decorridos oito dias após a infestação, avaliou-se a mortalidade dos carunchos por meio da contagem direta do número de adultos mortos presentes nas repetições. Os insetos remanescentes foram retirados e descartados, deixando apenas os grãos infestados com os ovos.

Por ocasião da avaliação da mortalidade dos adultos, também se realizou a contagem dos ovos por grão. Essa contagem foi feita com o auxílio de uma lupa de bolso de 10x de aumento. Em seguida, os grãos foram colocados novamente nos copos e mantidos no laboratório de Entomologia em condições de ambiente, com uma temperatura média de $28,8{ }^{\circ} \mathrm{C}$, umidade relativa de $60,5 \%$ e fotofase de 14 horas.

\subsection{Efeito sobre a emergência dos insetos da geração F1}

Após quarenta e cinco dias da infestação, tempo necessário para que a progênie dos insetos completasse seu ciclo e atuassem sobre a massa de grãos, avaliaram-se o número de insetos emergidos em cada repetição, matando-os com a utilização de formol embebido em um chumaço de algodão o qual foi colocado sob as tampas e, em seguida, colocou-se sobre os copos de acrílicos com os insetos. 


\subsection{Efeito sobre a perda de peso dos grãos ocasionada pelas larvas}

Depois de realizar as avaliações da emergência, fez-se a pesagem dos grãos em uma balança analítica para determinar a perda de peso provocada pela alimentação das larvas do inseto no interior dos grãos.

A determinação da perda de peso foi feita fazendo-se a pesagem no início da infestação e depois se pesando no final do experimento (45 dias). A perda foi obtida subtraindo-se o peso final do peso inicial dos 50 grãos contidos nos copos de acrílico.

Os dados obtidos no experimento foram submetidos à análise de variância e as suas médias comparadas pelo teste de Tukey $(P \leq 0,05)$.

\section{RESULTADOS E DISCUSSÃO}

\subsection{Efeito sobre a mortalidade e oviposição dos adultos}

Não houve interação significativa entre as cultivares de feijão e as condições atmosféricas para mortalidade e oviposição dos adultos. Analisando-se os fatores isoladamente, percebeu-se que os insetos que foram expostos ao feijão caupi, apresentaram menor mortalidade (60\%), quando comparado com o feijão carioca, que causou uma mortalidade de 90,45\% (Tabela 1).

\section{Tabela 1: Porcentagem de mortalidade de adultos e número médio de ovos/grão (oviposição) de Callosobrucus maculatus após oito dias de infestação em duas cultivares de feijão e três condições atmosféricas a $28,8 \pm 1^{\circ} \mathrm{C}$, umidade relativa de 60,5 $\pm 10 \%$ e fotofase de 14 horas, Crato -2008 .}

\begin{tabular}{lcc}
\hline & \multicolumn{2}{c}{ Parâmetros Biológicos Avaliados } \\
\hline Cultivares de feijão & Mortalidade (\%) & Oviposição \\
Caupi & $60,00 \mathrm{a}$ & $15,76 \mathrm{a}$ \\
Carioca & $90,45 \mathrm{~b}$ & $9,74 \mathrm{~b}$ \\
F (Tratamento) & $5,54 * *$ & $4,46 * *$ \\
C.V. (\%) & 10,23 & 23,76 \\
\hline Condições atmosféricas & & \\
\hline Hermética & $92,90 \mathrm{a}$ & $9,15 \mathrm{a}$ \\
Semi-hermética & $92,10 \mathrm{a}$ & $10,75 \mathrm{a}$ \\
Não hermética & $80,00 \mathrm{~b}$ & $10,26 \mathrm{a}$ \\
F (Tratamento) & $5,54 \mathrm{~ns}$ & $4,46 \mathrm{~ns}$ \\
C.V. (\%) & 10,23 & 23,76 \\
\hline \multicolumn{2}{c}{${ }^{1}$ Médias seguidas pela mesma letra nas colunas não diferem entre si pelo teste de Tukey (P $\left.\leq 0,05\right)}$.
\end{tabular}

Como os carunchos têm uma longevidade de 10 dias e não se alimentam na fase adulta, acreditamos que a mortalidade não é um parâmetro adequado para avaliação do efeito dos grãos sobre o inseto e principalmente pelo tempo (oito dias) relativamente elevado, o que explica a alta mortalidade mesmo no caupi, onde o inseto é uma praga de importância econômica.

Inversamente proporcional a mortalidade, constatou-se que aqueles insetos que foram mantidos no feijão caupi, colocaram mais ovos por grão $(15,76)$, quando comparado com o feijão carioca $(9,74)$, que foi menos preferido para oviposição (Tabela 1). 
De acordo com Throne et al. (2000), diferentes fatores químicos podem estar intimamente relacionados com o gérmen dos grãos, conferindo implicações durante a oviposição. O caupi provavelmente libera algum cairomônio, sendo mais preferido para oviposição, ocorrendo o contrário para o carioca, o qual mesmo sendo um grão não hospedeiro do caruncho foi ovipositado.

Boiça Júnior et al. (2002) também verificaram menor oviposição de Zabrotes subfasciatus (Boh.) no feijão carioca em condições de laboratório em teste sem chance de escolha. Condições essas que foram dadas ao caruncho na presente pesquisa. Portanto, quando o inseto não tem chance de escolha, oviposita nos grãos do feijão carioca, mesmo não sendo um hospedeiro da praga.

Com relação às condições atmosféricas, percebeu-se maior mortalidade para as condições semi herméticas e herméticas (Tabela 1), não houve diferenças estatísticas na oviposição. Os adultos são muito sensíveis a baixas concentrações de oxigênio, pois precisam desse gás para a sua respiração e sobrevivência na massa de grãos (MBATA et al., 2005).

Mbata et al. (2005) verificaram que uma pressão de 1 bar a menos de 2 horas, causa 99\% de mortalidade dos adultos desse caruncho em grãos de caupi. A uma concentração de $4 \%$ de $\mathrm{O}_{2}$ e $20 \%$ de $\mathrm{CO}_{2}$, Conyers e Bell (2007) verificaram uma mortalidade de $99,4 \%$ de Sitophilus oryzae em grãos de arroz obtida durante vinte e oito dias de armazenamento.

Já Rozado et al. (2008) verificaram que o aumento do período de exposição do ozônio $\left(\mathrm{O}_{3}\right)$ aumenta a mortalidade de $S$. zeamais em grãos de milho. Em 2 horas e a uma pressão de 8 bar, Noomhorm et al. (2009) conseguiram obter $99 \%$ de mortalidade desse caruncho em grãos de milheto.

Como a pesquisa foi conduzida em condições semelhantes às encontradas na agricultura familiar em que os pequenos produtores não dispõem de equipamentos de medição dos gases atmosféricos, não foi possível medir as concentrações de $\mathrm{O}_{2}$ e $\mathrm{CO}_{2}$, dificultando a determinação da real relação entre a mortalidade dos adultos e a concentração dos gases.

Mas, de uma forma prática, o que na verdade é mais válido, é que a mortalidade dos carunchos aumenta com a diminuição do oxigênio e aumento de dióxido de carbono na massa de grãos.

Para os ovos, por serem mais tolerantes às condições herméticas, em virtude da proteção do cório, observou-se que a não diferença estatística ficou mais evidente nos tratamentos avaliados. Adler (1994) ao realizar uma pesquisa com o caruncho do gênero Sitophilus em milho constatou que o estádio de ovo é um dos mais tolerantes quando expostos a diferentes concentrações em atmosfera modificada.

De acordo com Afonso et al. (2000), quando elevamos o $\mathrm{CO}_{2}$ nos espaços intergranulares nas infestações contendo insetos em diversos estádios de desenvolvimento, principalmente ovos, a maior parte deles morrem quando a concentração de $\mathrm{O}_{2}$ é reduzida.

A uma concentração de $1 \%$ de $\mathrm{O}_{2}$ e $70 \%$ de $\mathrm{CO}_{2}$, Ofuya e Reichmuth (2002) evidenciaram uma maior mortalidade dos ovos de C. maculatus. Shazali et al. (2004) verificaram que ovos de um dia desse caruncho são os mais resistentes e para exterminá-lo na massa de grãos de feijão é preciso aplicar uma pressão de 30 bar durante 20 minutos. 
Mbata et al. (2004) constataram que os ovos de Plodia interpunctella (Hubner), Tribolium castaneum (Herbst), Rhyzopertha dominica (F.) e Cadra cautela (Walker) também foram tolerantes às baixas concentrações de oxigênio.

Essa tolerância é porque os ovos metabolizam a uma taxa mais lenta do que os insetos adultos (MBATA, 2005). Mbata et al. (2009) também verificaram que com exceção dos ovos, as diferenças nos períodos de exposição requeridos para alcançar $100 \%$ de mortalidade de $C$. maculatus depende do estádio de desenvolvimento do inseto e da cultivar do feijão caupi.

Portanto, não importa se a cultivar é suscetível ou não hospedeira, pois o inseto oviposita assim mesmo, independente das condições atmosféricas, já que os ovos são tolerantes a essas condições.

\subsection{Efeito sobre a emergência de insetos da geração F1}

Foi verificada interação significativa entre as cultivares de feijão e as condições atmosféricas para emergência dos insetos. Desdobrando a interação cultivares dentro de condições atmosféricas, observou-se que não houve emergência de insetos na cultivar carioca nas três condições atmosféricas (Tabela 2).

Apesar dos insetos chegarem a colocar ovos $(9,74)$ (Tabela 1$)$, as larvas após a eclosão provavelmente ao se alimentarem desse grão, morreram devido à presença de algum composto químico desfavorável ao desenvolvimento do inseto, não dando origem a nenhum adulto, já que esse grão não é hospedeiro do caruncho.

Tabela 2: Emergência de Callosobrucus maculatus em duas cultivares de feijão depois de quarenta e cinco dias de armazenamento em três condições atmosféricas, Crato-2008.

\begin{tabular}{lccc}
\hline Cultivares de feijão & \multicolumn{3}{c}{ Condições atmosféricas } \\
\cline { 2 - 4 } & Hermética & Semi-hermética & Não hermética \\
\hline Caupi & $223,5 \mathrm{Ab}$ & $387 \mathrm{Aa}$ & $453,75 \mathrm{Aa}$ \\
Carioca & $0,00 \mathrm{Ba}$ & $0,00 \mathrm{Ba}$ & $0,00 \mathrm{Ba}$ \\
\hline
\end{tabular}

${ }^{1}$ Médias seguidas pela mesma letra maiúscula nas colunas ou pela mesma letra minúscula nas linhas não diferem entre si pelo teste de Tukey $(P \leq 0,05), C . V .18,27$. Para análise os dados foram transformados em $\sqrt{ } x+1$.

A alta suscetibilidade de larvas de bruquídeos mantidas em cultivares resistentes de feijão e em baixas concentrações de oxigênio deve-se ao fato que as larvas alimentam-se ativamente e as cultivares resistentes contêm enzimas inibidoras como as inibidoras de tripsina (GATEHOUSE et al.,1979), inibidoras de $\alpha$-amilase (ISHIMOTO; CHRISPEELS, 1996) ou antimetabólitos presentes no cotilédone dos feijões (BIRCH et al., 1985).

As enzimas inibidoras ou os antimetabólitos impedem o desenvolvimento das larvas ou produzem larvas menos vigorosas e mais suscetíveis a baixas concentrações de oxigênio do que aquelas mantidas em cultivares menos suscetível.

Certamente a cultivar carioca apresentou em seus grãos algumas dessas enzimas ou antimetabólitos que impediram o desenvolvimento das larvas e consequentemente a emergência do C. maculatus. Boiça Junior et al. (2002) constataram emergência de Z. subfasciatus em grãos da cultivar carioca em condições de laboratório, o que demonstra a não preferência do $C$. maculatus por essa cultivar quando comparado com outros bruquídeos. 
No caupi houve uma maior emergência de insetos na condição não hermética com 453,75 insetos e na semi hermética, com 387 (Tabela 2), não diferindo estatisticamente entre si. Nessas mesmas condições, Sousa et al. (2006) também verificaram maior emergência de $S$. zeamais quando utilizaram a cultivar de milho milhometro.

Na condição hermética houve menor emergência, com apenas 223,5 insetos, ou seja, menos da metade, quando comparado com as outras condições atmosféricas. Com isso, torna-se evidente o efeito da interação, pois mesmo uma cultivar suscetível, quando submetida a uma condição hermética, consegue tolerar o ataque da praga dando origem a um menor número de insetos na massa de grãos.

O fato dos insetos não se desenvolverem bem nessas condições está relacionado a uma maior concentração de dióxido de carbono que é tóxico no seu estado normal e por ser um poderoso anestésico (FARONI et al., 2002).

Num sistema hermeticamente fechado, o teor de oxigênio é reduzido a um valor muito baixo, criando-se uma condição de anoxia e o teor de dióxido de carbono elevado a um nível onde a respiração cessa, deixando o ambiente na condição de hipercarbia (BEM et al., 2006), durante um longo período de armazenamento, condição semelhante a que ocorreu na presente pesquisa.

Sousa et al. (2006) observaram que a cultivar cruzeta de milho após ser armazenada por sessenta dias em condição hermética, reduziu a emergência de S. zeamais. Conyers e Bell (2007) eliminaram a emergência de $S$. granarius (L.) após vinte e oito dias de armazenamento de grãos de trigo a uma concentração de $20 \%$ de $\mathrm{CO}_{2}$ e $5 \%$ de $\mathrm{O}_{2}$.

Ao contrário, em condições não herméticas de armazenamento, Resende et al. (2008) observaram que a infestação de grãos de feijão pelo gorgulho Acanthoscelides obtectus (Say) cresce exponencialmente ao longo do armazenamento, pois nessas condições o oxigênio circula livremente entre os grãos.

\subsection{Efeito sobre a perda de peso dos grãos ocasionada pelas larvas}

Também foi verificada interação significativa entre as cultivares de feijão e as condições atmosféricas para perda de peso dos grãos. Desdobrando a interação cultivares dentro de condições atmosféricas, observou-se que não houve diferenças significativas na cultivar carioca (Tabela 3). Como não houve emergência de carunchos nessa cultivar (Tabela 2), era de se esperar também que não houvesse perdas de pesos, pois as larvas nem chegaram a penetrar nos grãos.

Já na cultivar caupi, a condição hermética proporcionou a menor perda de peso $(2,61 \mathrm{~g})$, quando comparado com as condições semi-hermética e não hermética que não diferiram estatisticamente entre si e provocaram perdas maiores, da ordem de 4,10 e 4,56g, respectivamente, ou seja, praticamente o dobro.

Com isso, constata-se que nas condições em que ocorre circulação de oxigênio, há um maior consumo dos grãos pelas larvas. Sousa et al (2006) também constataram uma menor perda de peso em grãos de milho da cultivar cruzeta provocado pelo caruncho $S$. zeamais quando eles foram armazenados na condição hermética. 
Tabela 3: Perda de peso de grãos (g) nas duas cultivares de feijão infestadas com Callosobrucus maculatus depois de quarenta e cinco dias de armazenamento em três condições atmosféricas, Crato - 2008.

\begin{tabular}{lccc}
\hline Cultivares de feijão & \multicolumn{3}{c}{ Condições atmosféricas } \\
\cline { 2 - 4 } & Hermética & Semi-hermética & Não hermética \\
\hline Caupi & $2,61 \mathrm{Ab}$ & $4,10 \mathrm{Aa}$ & $4,56 \mathrm{Aa}$ \\
Carioca & $0,44 \mathrm{Ba}$ & $0,48 \mathrm{Ba}$ & $0,55 \mathrm{Ba}$ \\
\hline
\end{tabular}

${ }^{1}$ Médias seguidas pela mesma letra maiúscula nas colunas ou pela mesma letra minúscula nas linhas não diferem entre si pelo teste de Tukey $(P \leq 0,05)$. C.V. 30,18

A não preferência pela cultivar carioca pode estar associada a antixenose e/ou antibiose, pois não emergiram adultos, assim, algum fator do grão pode ter afetado o desenvolvimento impedindo a emergência no final do período de armazenamento. Isso explica a reduzida perda de peso nesses grãos que foi detectada devido às perdas de umidade dos grãos durante o período em que ficaram armazenados.

Guzzo et al. (2002) em experimentos com diferentes materiais de milho, também verificaram diferenças de consumo pelo $S$. zeamais entre os genótipos investigados, donde associaram a perda total de grãos ao baixo número de adultos emergidos.

Esse estudo contribui para trazer informações práticas para o pequeno produtor que poderão ser utilizadas na adoção do controle hermético em associação com cultivares suscetíveis de feijões no manejo comercial de carunchos em grãos armazenados, como uma alternativa ao uso do brometo de metila ou da fosfina.

Esses produtos podem trazer sérias implicações para a saúde dos consumidores e, hoje em dia, a população mundial busca produtos alimentares livres de inseticidas ou com pelo menos reduzido nível de resíduos.

Essa tecnologia será útil para grãos secos onde a fumigação com esses produtos têm se tornado uma ferramenta indispensável na proteção dos grãos contra a infestação de bruquídeos (MBATA et al., 2004).

\section{CONCLUSÃO}

A interação de uma cultivar suscetível de feijão armazenada em uma condição hermética pode ser utilizada como uma tática alternativa para o agricultor familiar ao uso do controle químico em programas de manejo comercial de Callosobruchus maculatus em grãos armazenados.

\section{REFERÊNCIAS BIBLIOGRÁFICAS}

1. ADLER, C. A. Compararison of the efficacy of $\mathrm{CO}_{2}$ rich and $\mathrm{N}_{2}$ rich atmospheres against the granary weevil Sitophilus granarius. In: INTERNATIONAL WORKING CONFERENCE ON STORED PROTECTION PRODUCT, 6, 1994, Wallingford. Proceedings...Wallingford: CAB International, 1994. p. 11-15.

2. AFONSO, A. D. L.; SILVA, J. S.; BERBERT, P. A. Controle de pragas por atmosferas controladas. In: SILVA, J. S. (Ed.). Secagem de produtos agrícolas. Viçosa: Aprenda Fácil, 2000. p. 383-393. 
3. BEM, D. C.; LIEM, P. V.; GUMMERT, M.; RICKMAN, J. F. Effect of hermetic storage in the super bag on seed quality and milled rice quality of different varieties in Bac Lieu,Vietnam. In: INTERNATIONAL RICE CONGRESS, 2, 2006, New Delhi. Resumos... New Delhi: IRRI, 2006. p. 567.

4. BIRCH, N.; SOUTHGATE, B. J.; FELLOWS, L. E. Wild and semi-cultivated legumes as potential sources of resistance to bruchid beetles for crop breeder: a study of Vigna/Phaseolus. In: DICKIE, J. B.; PRITCHARD, H. W.; PROBERT, R. J. (Eds). Plants for arid lands. London: Royal Botanic Gardens, 1985. p. 303-320.

5. BOIÇA JÚNIOR, A. L.; BOTELHO, A. C. G.; TOSCANO, L. C. Comportamento de genótipos de feijoeiro ao ataque de Zabrotes subfasciatus (Boheman, 18) (Coleoptera: Bruchidae) em condições de laboratório. Arquivos do Instituto Biológico de São Paulo, São Paulo, v. 69, n. 2, p. 51-55, 2002.

6. CONYERS, S. T.; BELL, C. H. A novel use of modified atmospheres: storage insect population control. Journal of Stored Products Research, Oxford, v. 43, p. 367-374, 2007.

7. FARONI, L. R.; GUEDES, D. A.; BERBEERT, R. N. C.; SILVA, A. P. R. A. Atmosfera modificada no controle das pragas de grãos armazenados. In: LORINI, I.; MIIKE, L. H.; SCUSSEL, V. M. (Eds). Armazenagem de grãos. Vol. 1. Campinas: IBG, 2002. p. 463-491.

8. GALLO, D.; NAKANO, O.; SILVEIRA NETO, S.; CARVALHO, R. P. L.; BAPTISTA, G. C. de.; BERTI FILHO, E.; PARRA, J. R. P.; ZUCCHI, R. A.; ALVES, S. B.; VENDRAMIM, J. D.; MARCHINI, L. C.; LOPES, J. R. S.; OMOTO, C. Entomologia Agrícola. Piracicaba: FEALQ, 2002. 920 p.

9. Gatehouse, A. M. R.; GATehouse, J. A.; DOBIE, P.; KILMINSTER, A. M.; BOULTER D. Biochemical basis of insect resistance in Vigna unguiculata. Journal of the Science of Food and Agriculture, DAVIS, v. 30, p. 949-958, 1979.

10. GUZZO, E. C.; ALVES, L. F. A.; ZANIN, A.; VENDRAMIN, J. D. Identificação de materiais de milho resistentes ao ataque de gorgulho Sitophilus zeamais (Mots., 1855) (Coleoptera: Curculionidae). Arquivos do Instituto Biológico de São Paulo, São Paulo, v. 69, n. 2, p. 69-73, 2002.

11. ISHIMOTO, M.; CHRISPEELS, M. J. Protective mechanism of the Mexican bean weevil against high levels of $\alpha$-amylase inhibitor in the common bean. Plant Physiology, Maryland, v. 111, p. 393-401, 1996.

12. MACEDO, M. L. R.; FREIRE, M. G. M.; NOVELLO, J. C.; MARANGONI, S. Talisia esculenta lectin and larval development of Callosobruchus maculates and Zabrotes subfasciatus (Coleoptera: Bruchidae). Biochimica et Biophysica Acta, Amsterdam, n. 1571, p. 83-88, 2002.

13. MBATA, G. N.; JOHNSON, M.; PHILLIPS, T. W.; PAYTON, M. Mortality of life stages of cowpea weevil (Coleoptera: Bruchidae) exposed to low pressure at different temperatures. Journal of Economic Entomology, Riverside, v. 98, n. 3, p. 1070-1075, 2005.

14. MBATA, G. N.; PHILLIPS, T. W. Effects of temperature and exposure time on mortality of stored-product insects exposed to low pressure. Journal of Economic Entomology, Riverside, v. 94, p. 1302-1307, 2001. 
15. MBATA, G. N.; PHILLIPS, T. W.; PAYTON, M. Mortality of eggs of stored-product insect held under vacuum: effects of pressure, temperature, and exposure time. Journal of Economic Entomology, Riverside, v. 97, p. 353-360, 2004.

16. MBATA, G. N.; PHILLIPS, T. W.; PAYTON, M. E. Effects of cowpea varietal susceptibility and low pressure on the mortality of life stages of Callosobruchus maculatus (Coleoptera: Bruchidae). Journal of Stored Products Research, Oxford, v. 45, p. 232-235, 2009.

17. NOOMHORM, A.; SIRISOONTARALAK, P.; URAICHUEN, J.; AHMAD, I. Effects of pressurized carbon dioxide on controlling Sitophilus zeamais (Coleoptera: Curculionidae) and the quality of milled rice. Journal of Stored Products Research, Oxford, v. 45, p. 201-205, 2009.

18. OFUYA, T. I.; REICHMUTH, C. Effect of relative humidity on the susceptibility of Callosobruchus maculatus (Fabricius) (Coleoptera: Bruchidae) to two modified atmospheres. Journal of Stored Products Research, Oxford, v. 38, p. 139-146, 2002.

19. PADIN, S.; BELLO, G. D.; FABRIZIO, M. Grain loss caused by Tribolium castaneum, Sitophilus oryzae and Acanthoscelides obtectus in stored durum wheat and beans treated with Beauveria bassiana. Journal of Stored Products Research, Oxford, v. 38, p. 69-74, 2002.

20. RESENDE, O.; CORRÊA, P. C.; FARONI, L. R. A.; CECON, P. R. Avaliação da qualidade tecnológica do feijão durante o armazenamento. Ciência e Agrotecnologia, Lavras, v. 32, n. 2, p. 517524, 2008.

21. ROZADO, A. F.; FARONI, L. R. A.; URRUCHI, W. M. I.; GUEDES, R. N. C; PAES, J. L. Aplicação de ozônio contra Sitophilus zeamais e Tribolium castaneum em milho armazenado. Revista Brasileira de Engenharia Agrícola e Ambiental, Campina Grande, v. 12, n. 3, p. 282-285, 2008.

22. SHAZALI, M. E. H.; IMAMURA, T.; MIYANOSHITA, A. Mortality of eggs of the cowpea bruchid, Callosobrucus maculatus (F.) (Coleoptera: Bruchidae) in carbon dioxide under high pressure. Applied Entomology Zoology, v. 39, n. 1, p. 49-53, 2004.

23. SOUSA, A. H.; MARACAJÁ, P. B.; COSTA, A. A.; GIRALDO, A. S.; PEREIRA, T. F. Desempeño de Sitophilus zeamais (Coleoptera: Curculionidae) em diferentes variedades de maíz y condiciones atmosféricas. Revista Verde, Mossoró, v. 1, n. 1, p.20-25, 2006.

24. THRONE, J. E.; BAKER, J. E.; MESSI-NA, F. J.; KRAMER, K. J.; HOWARD, J. A. Varietal resistance. In: SUBRAMANYAM, B.; HAGSTRUM, D. W. (Eds). Alternatives to pesticides in storedproduct. Vol. 1. Massachusetts: Kluwer Academic Massachusetts, 2000, p. 165-192. 